\title{
Design of Biometric Authentication System using Three Basic Human Traits
}

\author{
Palvi Sharma ${ }^{1}$, Manit Kapoor ${ }^{2}$, Dr. Naveen Dhillon ${ }^{3}$ \\ ${ }^{1}$ M-TECH Student, R.I.E.T Phagwara \\ ${ }^{2}$ Assistant Professor, R.I.E.T Phagwara \\ ${ }^{3}$ Professor, RIET Phagwara
}

\begin{abstract}
Todays the single biometrics feature is not sufficient to provide secure authentication. Most of the multi-modal techniques are lacking in security aspect. Biometric system utilize in various system for the identification or authentication approval. Biometric authentication system utilizes various biometric traits for the matching between various biometric traits. These feature values that have been computed by using various approaches for biometric traits like face, finger and iris. These feature value has been stored in database for matching purpose using distance classifier. After fusion these values has been computed for all the images present in the database and then test images has been selected and undergoes matching process on the basis of distance classifier. Min distance computed represents the maximum matching of the feature of different biometric traits. Parameter selected for the performance evolution of proposed system are FAR (False Acceptance Rate), FRR (False Rejection Rate) and ACCURACY.
\end{abstract}

Keywords: Bio-metrics, Multi-Thresholding

\section{Introduction}

Biometrics is combination of two Greek words Bios (life) and metrikos (measure). It is recognized that some human body characteristics such as face, gait or voice can be used to distinguish individual from a group of people. In a biometrics system a person is recognized on the basis of physical and behavioural traits. In it pattern recognition is used. In the process of pattern recognition human traits are captured on and then matched with the database. Biometric identifiers are the unique, measurable qualities used to mark and depict individuals. Biometric identifiers are regularly sorted as physiological versus behavioural characteristics. Physiological attributes are identified with the state of the body. Samples incorporate, however are not constrained to unique finger impression, palm veins, face recognition, DNA, palm print, hand geometry, iris distinguishment, retina and smell/fragrance. Behavioural attributes are identified with the example of conduct of an individual, including however not constrained to writing mood, walk, and voice. The primary thoughts of biometrics seemed numerous years back. By and large, it is extremely hard to say that biometrics showed up it this spot at this point. The thoughts to utilize parts of human body and even the approaches to utilize this thoughts seemed everywhere throughout the world. In the first place confirmations of biometrics showed up in 29.000 bc when the cave dwellers utilized their fingerprints to sign their drawings. The initially recorded proof of utilizing biometric confirmation was as a part of old Egypt [11].

One of the executives, amid the development of extraordinary pyramid of Khufu, attempted to systemize the methodology of giving sustenance to specialists. He recorded all data about the specialist (name, age, work unit, position, occupation, and so on). Anyway afterward that numerous specialists swindled him, the head started to record the physical and behavioural qualities. In fourteenth century in China biometric confirmation was somewhat prominent among vendors [6].

Innovation of ahead of schedule biometrics was fairly straightforward: paper with ink permitted to take palm print $\mathrm{s}$ and foot shaped impressions of youngsters so as to separate them from other. It is fascinating to bring up that despite its effortlessness thusly of bimetric validation is still being used and is the most popular.

\section{Characteristics of Biometrics}

Any physical and/or behaviour characteristics of a human can be considered as a biometric if it exhibits following characteristics:

- Universality: Each person accessing the biometric application should posses a valid biometric trait.

- Uniqueness: The given biometric trait should exhibits distinct features across individuals comprising the population.

- Permanence: The biometric characteristics should remain sufficient invariant over a period of time.

- Measurability: The biometric characteristics can be quantitatively measured i.e. acquiring and processing of biometric trait should not cause inconvenience to the individual.

- Performance: The biometric trait should the required accuracy imposed by the application[12]

\section{Types of Biometric Systems}

Biometric system is broadly categorized in two types: Physical and Behavioural [7] (Jain et al. 2010):

Physical Biometrics

Finger print Recognition

Facial Recognition

Hand Geometry 
IRIS Recognition

DNA

Behavioral Biometrics

Speaker Recognition

Signature

Keystroke

Walking style

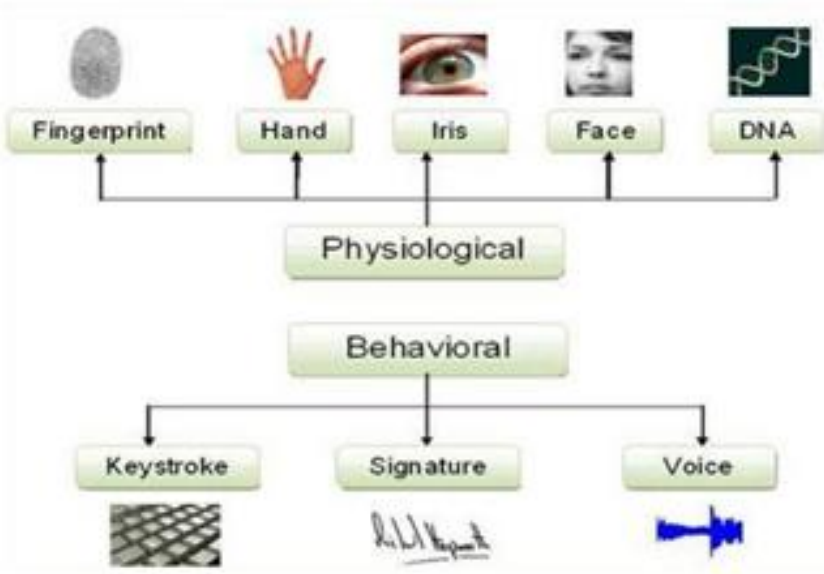

Figure 1:Types of Biometrics

\section{Operations of a Biometric System}

Based on the appliance, a biometric system may operate either in verification mode or identification mode [4] (Jain et al. 2004),In the verification mode, a person's identity is validated through comparison between the captured biometric data and its own biometric templates stored in the database of the system. In such a system, an individual who is desirable of recognition gives a claim of identity, usually a $\mathrm{PIN}$ and a one-to-one comparison is done by the system to determine if claim is true or not [8] (Wayman 2001). In the identification mode, an individual is recognised by questing through the templates (stored in database) of all the users for a good match. Therefore, a one-tomany comparison is made by the system to establish the identity of a user (or negative response is given if the subject is not part of the system's database) without a subject having to put the efforts for claiming his/her identity[8](Wayman2001).

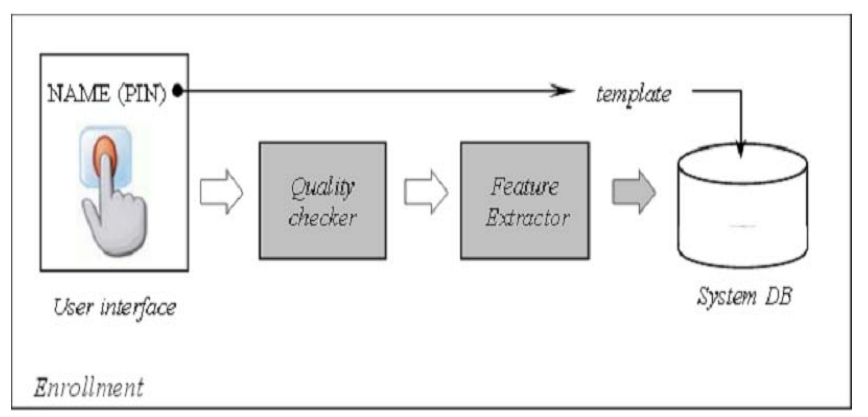

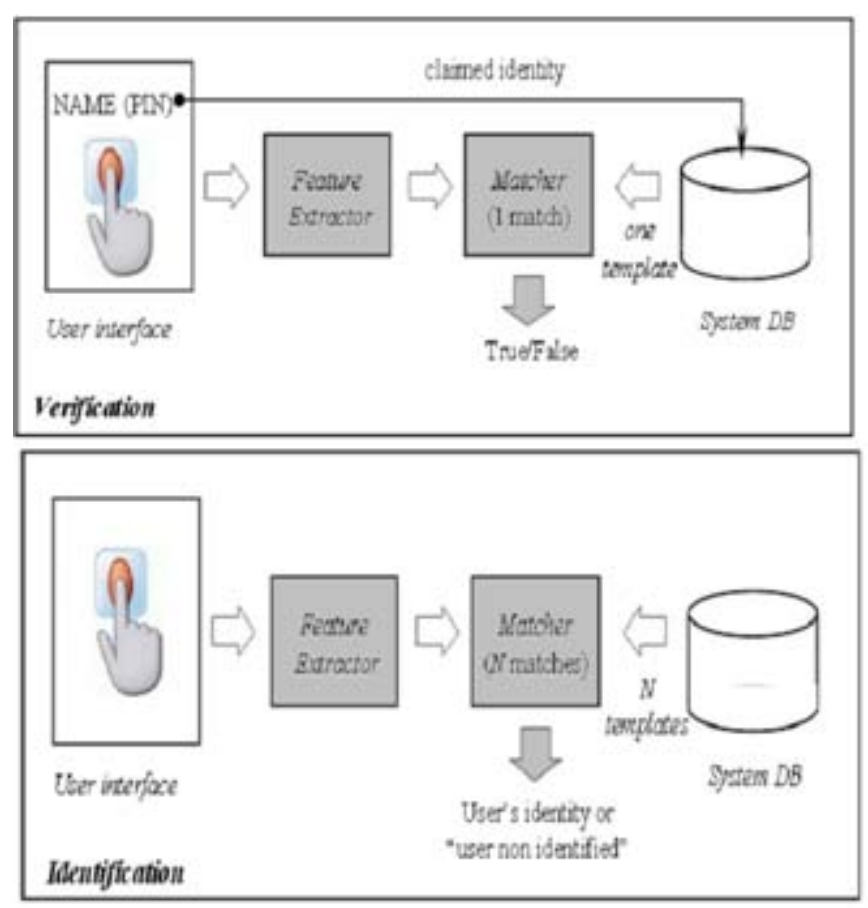

Figure 2: Block diagrams of enrolment, verification and identification tasks are shown using the four main modules of a biometric system, i.e., sensor, feature extractor, matcher, and system database.

\section{Methodology}

The whole process can be shown in the form of a flow diagram:

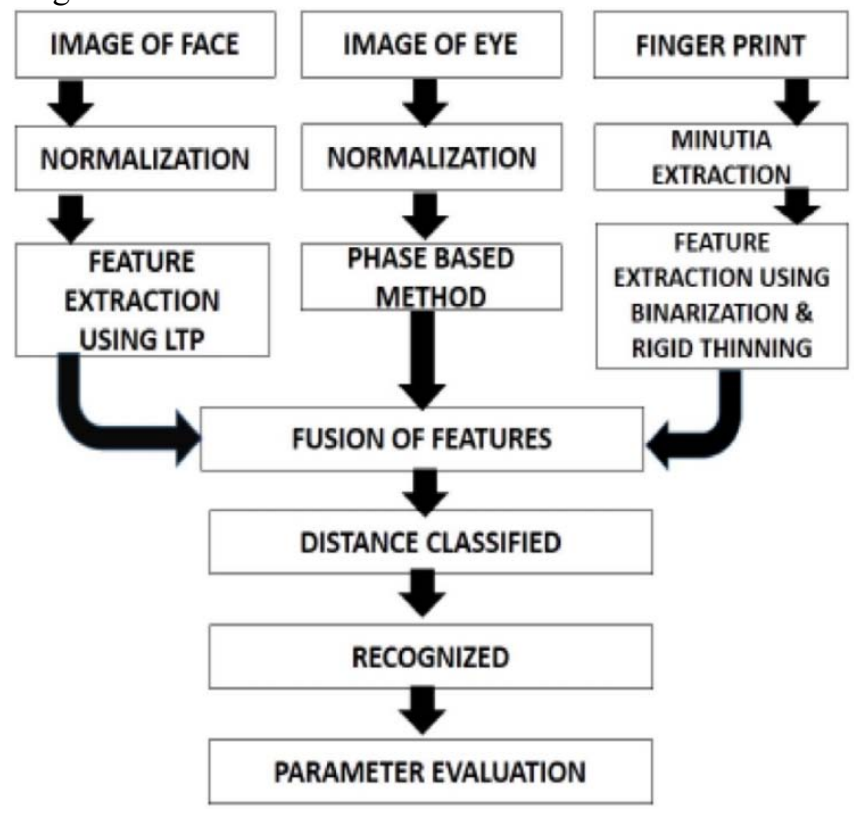

Figure 3: Flow Chart of the system

This paper presents a multimodal biometric system comprising the fusion of iris, fingerprint and face templates. The existence of iris and fingerprint images is firstly aggrandized using a series of pre-processing techniques which include segmentation andIris Images Fingerprint Images Face Images Pre-processing i.e. Segmentation \& Normalisation Pre-processing i.e. Segmentation \& Normalisation Feature Extraction Feature Extraction Feature Extraction Information Fusion Calculation of Scores for 


\section{International Journal of Science and Research (IJSR) \\ ISSN (Online): 2319-7064}

Index Copernicus Value (2013): 6.14 | Impact Factor (2014): 5.611

verifying the Genuine \& the Imposter Validation/Classification. Below are ten data base images for the IRIS that we have selected for the system:

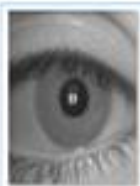

1

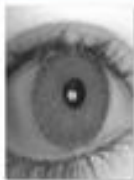

6

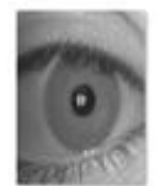

2

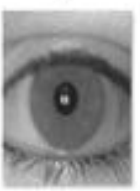

7

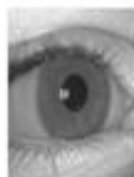

3

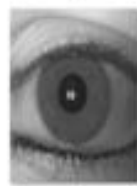

8

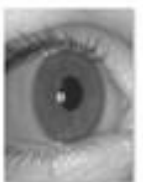

4

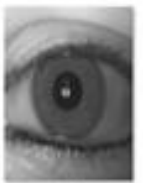

9

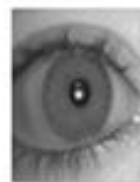

5

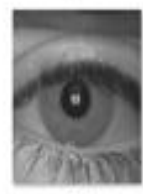

10
Figure 4: Ten images of IRIS data
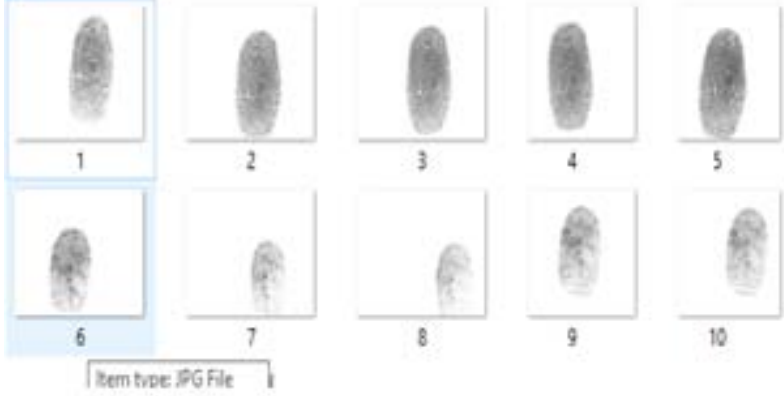

Figure 5: Fingerprint sample images
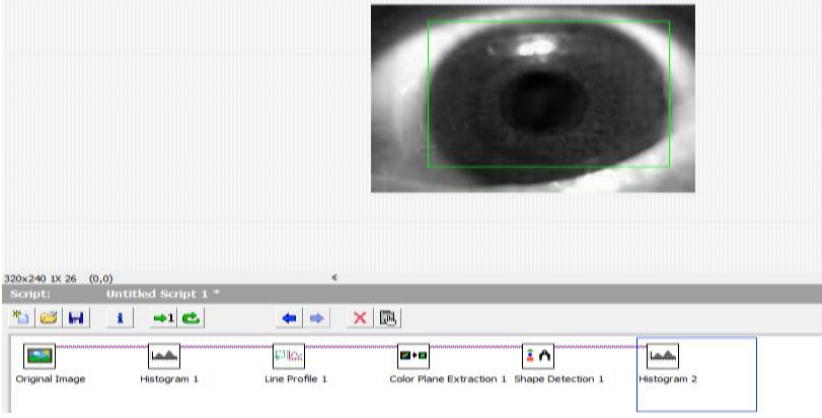

Figure 6: Input Iris Image for feature extraction

This figure represents the function of iris button. The image taken from the database for feature detection and histogram equalization

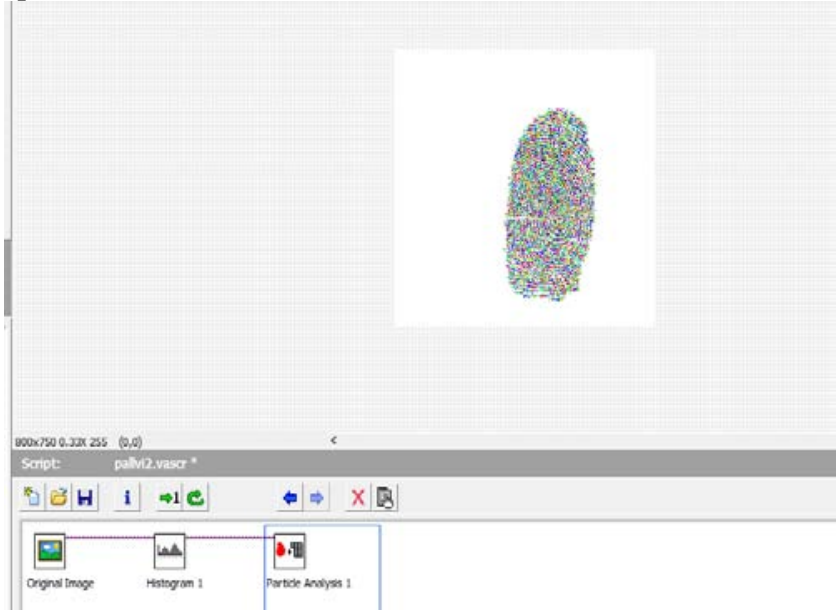

Figure 7: Input Finger Image for feature extraction

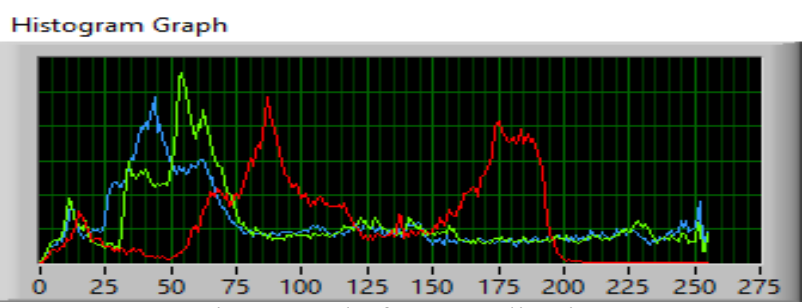

Figure 8: Histogram before equalization

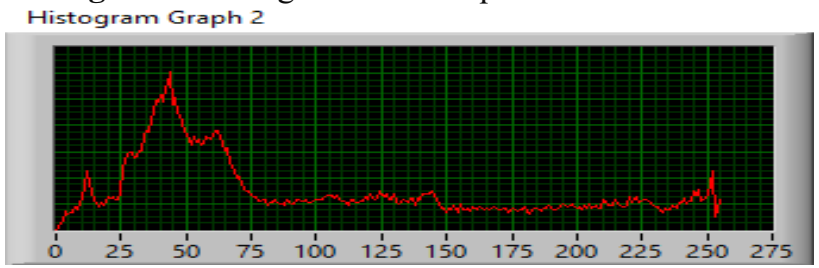

Figure 9: After equalization

\section{Results \& Discussion}

After extraction of features from various biometric traits fusion of feature on the basis of score level has to be done. Fusion techniques are divided into two different categories which are score level fusion and rank level fusion. In proposed work score level fusion approach has been utilized. After fusion the feature has to be saved in a file for recognition and authentication purpose. Next we have matching database. Once the enrolment of data is done by generating unique id and user id, now we only need to select your database then it will automatically extract the features and verify it with the stored data. If data matches with any of the stored data then the name of the user appears. Graphic user interface developed for the purpose of matching process which has to be performed on the database. In this various controls has been used for different purposes. This image is use to represents extraction of face features from the test face database. After extraction of face features fusion of the all iris, finger and face feature has to be done on the basis of score level fusion approach. Distance classification done with the database on the basis of different distance classifier. This classifier computes the distance between the different features of database images and to the query image features. These features described the similarity between different biometric traits. Where the minimum distance has been computed that is most matched feature vector. From that particular feature vector name of the identity is fetched out.

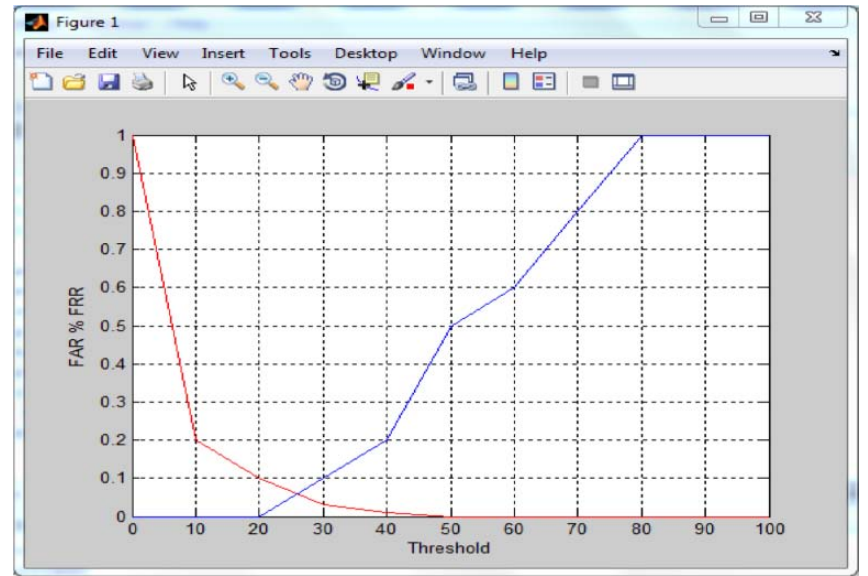

Figure 10: Graph FAR \& FRR Percentage 


\section{International Journal of Science and Research (IJSR) \\ ISSN (Online): 2319-7064}

Index Copernicus Value (2013): 6.14 | Impact Factor (2014): 5.611

This graph is use to represent the reliability of a system. Reliability is defined as the amount of time in which our system works properly. The point where the FAR and FRR curves meet is the equal error rate. It is the common alternative to define the performance of the system

Table displays experimental results for recognition with face image alone and with the fusion of all the three modalities i.e face, fingerprint and iris. The results are better when compared with the base paper as a new modality (iris) is added. A minimum FAR of $1.1 \%$ is attained as compare to $1.6 \%$ in case of face alone and maximum FAR is limited to $3.8 \%$ with the proposed methodology. The overall GAR\% achieved for the fusion of face, fingerprint and iris is $94 \%$ as compared to $89 \%$.

Table 4.1: Result Table

\begin{tabular}{|c|c|c|c|c|}
\hline Parameter & IRIS & Fingerprint & Face & Fusion \\
\hline FAR & 0.01 & 0.0037 & 0.1437 & 0.0148 \\
\hline FRR & 0.0021 & 0.91 & 0.79 & 0.98 \\
\hline GAR & .92 & .89 & .91 & 0.94 \\
\hline Area(PIXAL) & 76800 & 72416 & 75002 & 74608 \\
\hline SD & 0.9282 & 0.0579 & 0.0589 & 0.0085 \\
\hline Mean & 1.14 & 1.77 & 1.17 & 1.77 \\
\hline Min & .02 & 0.12 & 0.15 & 0.12 \\
\hline Max & 2.55 & 2.54 & 2.14 & 2.54 \\
\hline ERROR RATE & .991 & 0.384 & 0.422 & 0.099 \\
\hline
\end{tabular}

\section{Conclusion}

Biometric system utilize in various system for the identification or authentication approval. Biometric authentication system utilizes various biometric traits for the matching between various biometric traits. Various approaches have been used for the extraction of features from various types of biometric traits. In the proposed work the biometric traits utilize are face, fingerprint and iris. Single Biometric trait system is fail to provide accuracy for the authentication of different identities because due to single biometric trait the chances of mismatching increases. So to overcome these disadvantages of single trait biometric system, multimodal biometric system come into existence. Multimodal biometric system use face, finger and iris images for the development of proposed system. Feature from each biometric credential has been extracted and fused on the basis of score level fusion to reduce feature dimension. Computation speed increases due to reduction in feature dimension of fused features. This proposed system provides accuracy of $100 \%$. This provides better security than other biometric system because illegal availability of all the traits of single person is not available to match and perform any illegal operation. So one can conclude that multimodal biometric system provides better result as compare to single biometric trait system.

\section{References}

[1] Abdolahi Mohamad, Mohamadi Majid, Jafari Mehdi, “ Multimodal biometric system fusion using fingerprint and iris with fuzzy logic" International Journal of Soft Computing and Engineering (IJSCE) 2013, ISSN: 22312307 , Issue-6, pp. $504-510$
[2] Bansal Roli,Sehgal Priti and Bedi Punam, " Minutiae Extraction from Fingerprint Images- a Review," IJCSI International Journal of Computer Science Issues, September 2011, Vol. 8 Issue 5, No 3.

[3] Chaikan, P., Karnjanadecha, M., "A Reference Point Detection Algorithm for Top-View Finger Image Recognition" 5th International Symposium on Image and Signal Processing and Analysis, 2007,pp. 347 350 .

[4] Connaughton, R. Sgroi, A ; Bowyer, K.W. ; Flynn, P. "A cross-sensor evaluation of three commercial iris cameras for iris biometrics" IEEE Computer Society Conference on Computer Vision and Pattern Recognition Workshops (CVPRW), 2011 ,pp. 90 - 97.

[5] Cardoso, L. , Barbosa, A. ,Silva, F. , Pinheiro, A.M.G. "Iris Biometrics: Synthesis of Degraded Ocular Images" IEEE Transactions on Information Forensics and Security, Volume:8, pp. $1115-1125$.

[6] Deshmukh Amit,Pawar Sheetal,Joshi Dr. Madhuri, “ Feature level Fusion of Face and Fingerprint Modalities using Gabor Filter Bank” IEEE,2013

[7] Eshwarappa M.N,Dr Mrityunjaya V.Latte, “ Multimodal Biometric Person Authentication using Speech,Signature and Handwrititing Features,International journal of Advanced Computer Science and Applications,pp. 77 - 86

[8] Fernandez-Saavedra, B., Liu-Jimenez, J. ; SanchezAvila, C., "Quality Measurements for Iris Images in Biometrics". The International Conference on \&\#34; Computer as a Tool\&\#34 EUROCON, 2007, pp. $759-$ 764.

[9] Gonzalez R and Woods. R, Digital image processing ( $2^{\text {nd }}$ edition). Prentice-Hall, Englewood Cliffs, NJ, 2002. 1,2

[10] Chaikan, P., Karnjanadecha, M., "A Reference Point Detection Algorithm for Top-View Finger Image Recognition" 5th International Symposium on Image and Signal Processing and Analysis, 2007,pp. $347-$ 350 .

[11] Connaughton, R. Sgroi, A ; Bowyer, K.W. ; Flynn, P. "A cross-sensor evaluation of three commercial iris cameras for iris biometrics" IEEE Computer Society Conference on Computer Vision and Pattern Recognition Workshops (CVPRW), 2011 ,pp. 90 - 97.

[12] Cardoso, L. , Barbosa, A. ,Silva, F. , Pinheiro, A.M.G. "Iris Biometrics: Synthesis of Degraded Ocular Images" IEEE Transactions on Information Forensics and Security, Volume:8, pp. $1115-1125$.

[13] Deshmukh Amit,Pawar Sheetal,Joshi Dr. ]supervised support vector machines" 3rd IEEE International Conference on Computer Science and Information Technology (ICCSIT), 2010, pp. 267 - 270 ,vol. 3.

[14] Jani, R. Agrawal, N., "A Proposed Framework for Enhancing Security in Fingerprint and Finger-Vein Multimodal Biometric Recognition" International Conference on Machine Intelligence and Research Advancement (ICMIRA), 2013 , pp. 440 - 444.

[15] Kanade, S. , Camara, D. ; Krichen, E. ; PetrovskaDelacr.taz, D. "Three factor scheme for biometricbased cryptographic key regeneration using iris" Biometrics Symposium, 2008. BSYM '08, pp. 59- 64.

[16] Koneru. Anuradha, Tyagi Manoj Kumar, “ A Novel method for face recognition usingLbp,Ltp and Gabor 


\section{International Journal of Science and Research (IJSR) \\ ISSN (Online): 2319-7064}

Index Copernicus Value (2013): 6.14 | Impact Factor (2014): 5.611

features" International Journal of Scientific \& Technology Research , June 2012, VOLUME 1, ISSUE 5, ISSN 2277-8616 pp. 31-35

[17] Kekre H B, Bharadi V A," Ageing Adaptation for Multimodal Biometrics using Adaptive Feature Set Update Algorithm" IEEE International Advance Computing Conference (IACC)Patiala,India, 6-7 March 2009, pp.535-540

[18] The research and implementation of the method of pretreating the face images based on Open CV machine visual library" International Conference on Electronic and Mechanical Engineering and Information Technology (EMEIT), 2011, pp. 2719 - 2721, vol. 11.

[19] Ravi S, Mankame Dattatreya P., "Multimodal Biometric Approach Using Fingerprint Face and Enhanced Features Recognition" International Conerence on circuits,power and Computing Technologies[ICCPCT],IEEE 2013,PP. 1143-1149

[20] Shangling Song, Zhi Liu, “"An embedded real-time finger-vein recognition system for mobile devices" IEEE Transactions on Consumer Electronics, 2012, pp. $522-527$, vol. 58 .

[21] Weichun Cheng, Gaoyun An, "Face template protection using chaotic encryption" 5th IET International Conference on Wireless, Mobile and Multimedia Networks (ICWMMN 2013), pp. 245 - 248.

[22] Wan-Kou Yang, "Fuzzy inverse fisher discriminant analysis for face recognition"International Conference on Wavelet Analysis and Pattern Recognition, 2007, vol. 1, pp. $107-111$.

[23] Woodard, D.L., Pundlik, S ; Miller, P. ; Jillela, R. "On the Fusion of Periocular and Iris Biometrics in Nonideal Imagery" 20th International Conference on Pattern Recognition (ICPR), 2010,pp. 201 - 204.

[24] Wang Chuandong,Yang Yanying ,"Robust face recognition from single training image per person via auto-associative memory neural network" International Conference on Electrical and Control Engineering (ICECE),2011, pp. 4947 - 4950. 